\title{
Lactate dehydrogenase 5 : identification of a druggable target to reduce oxaluria
}

\author{
Jacob S. Stevens and Qais Al-Awqati \\ Department of Medicine, Division of Nephrology, Vagelos College of Physicians and Surgeons, Columbia University, New York, New York, USA.
}

\begin{abstract}
Excessive excretion of oxalate in the urine results in the formation of calcium oxalate crystals and subsequent kidney stone formation. Severe forms of hyperoxaluria, including genetic forms and those that result from ethylene glycol poisoning, can result in end-stage renal disease. Therapeutic interventions are limited and often rely on dietary intervention. In this issue of the $J C I$, Le Dudal and colleagues demonstrate that the lactate dehydrogenase 5 inhibitor (LDH5) stiripentol reduces urinary oxalate excretion. Importantly, stiripentol treatment of a single individual with primary hyperoxaluria reduced the urinary oxalate excretion. Together, these results support further evaluation of LDH5 as a therapeutic target for hyperoxaluria.
\end{abstract}

\section{Looking beyond diet to control oxaluria}

Oxalate $\left(\mathrm{C}_{2} \mathrm{O}_{4}{ }^{2-}\right)$, a final product of intermediary metabolism of several carbohydrates and amino acids, is largely produced by the liver and then excreted in the urine along with oxalate absorbed in the gut from dietary sources. Calcium oxalate in the urine is highly insoluble, but in healthy individuals, the concentration of this salt rarely, if ever, exceeds supersaturation. Yet calcium oxalate $(\mathrm{CaOx})$ stones occur in a substantial fraction of the world population with estimates of prevalence ranging from $3 \%$ to $5 \%$ of people worldwide. Oxaluria can result from multiple causes, including hyperabsorption syndromes and genetic causes of overproduction. Dietary control of oxaluria is the mainstay of therapy; however, this intervention is difficult and may not be sufficient to reduce urinary oxalate excretion. Unfortunately, other effective treatments are limited. In this issue, Le Dudal and colleagues describe a welcome serendipitous finding where a drug (stiripentol) that is used to treat a rare severe form of childhood epilepsy reduced urinary oxalate excretion, providing a pathway for pharmaceutical development (1).

Stiripentol, a drug used to treat the neuroepileptic disorder Dravet syndrome, was initially thought to directly enhance GABAergic neurotransmission (2). More compelling studies have shown that the drug inhibits lactate dehydrogenase (LDH), thereby interrupting the astrocyte-neuron lactate shuttle (3) where lactate, produced by astrocytes through the action of $\mathrm{LDH}$ isoenzyme 5 (LDH5), enters neurons and is converted to pyruvate by LDH1, and reduces the intracellular energy reliance on ketones. Lactate also signals by binding to neuronal GPCR HCAR1. Collectively, the astrocyte-neuron lactate shuttle and lactate action on HCAR1 result in hyperpolarization of neurons and a decreased number and duration of paroxysmal discharges (4). Stiripentol inhibits LDH5, with a half maximal concentration of about $500 \mu \mathrm{M}$ (3), which is close to what the recommended $50 \mathrm{mg} / \mathrm{kg}$ oral dose might produce in vivo, indicating a low specificity and affinity for its target.

Related Article: p. 2571

Conflict of interest: The authors have declared that no conflict of interest exists.

LDH5, an LDH tetrameter composed of 4 A-type subunits, is the isoenzyme expressed in muscle and importantly in the liver, where it controls hepatic oxalate production (Figure 1). Le Dudal et al. hypothesized that treatment with stiripentol might decrease hepatic oxalate production-both from endogenous and exogenous precursors-and thereby reduce urinary oxalate excretion (1). The authors showed that stiripentol, when administered to hepatocytes in vitro or given to healthy rats, decreased oxalate production and urinary excretion. When rats were poisoned with ethylene glycol, which induced severe hyperoxaluria, coadministration of stiripentol reduced $\mathrm{CaOx}$ crystal volume and renal failure. Further, in animals given a diet high in hydroxyproline, which increases oxalate production (Figure 1), stiripentol treatment increased urinary glycolate excretion, reduced urinary $\mathrm{CaOx}$ crystal volume, and limited renal dysfunction compared with rats receiving hydroxyproline diet alone. Most importantly, Le Dudal et al. administered stiripentol to a young woman with type I primary hyperoxaluria (AGT1 mutation) in graded doses up to $50 \mathrm{mg} / \mathrm{kg} / \mathrm{d}$ and found that her urinary oxalate-to-urinary creatinine ratio fell from $0.18 \mathrm{mmol}$ oxalate $/ \mathrm{mmol}$ creatinine to $0.068 \mathrm{mmol}$ oxalate $/ \mathrm{mmol}$ creatinine. In this multifaceted approach, the authors showed that stiripentol is effective in decreasing hyperoxaluria in various scenarios and preventing renal failure, a major longterm consequence of oxaluria. It is important to note that a $50 \%$ reduction in urinary oxalate concentration is usually enough to prevent the formation of $\mathrm{CaOx}$ stones.

\section{Implications of stiripentol for oxaluria}

A review of glyoxylate and oxalate metabolism and the exogenous and endogenous conditions that lead to increases in urinary $\mathrm{CaOx}$ concentrations is important to fully understand the implications of this new therapy for treating oxaluria (Figure 1) (5). Under normal physiologic conditions, 


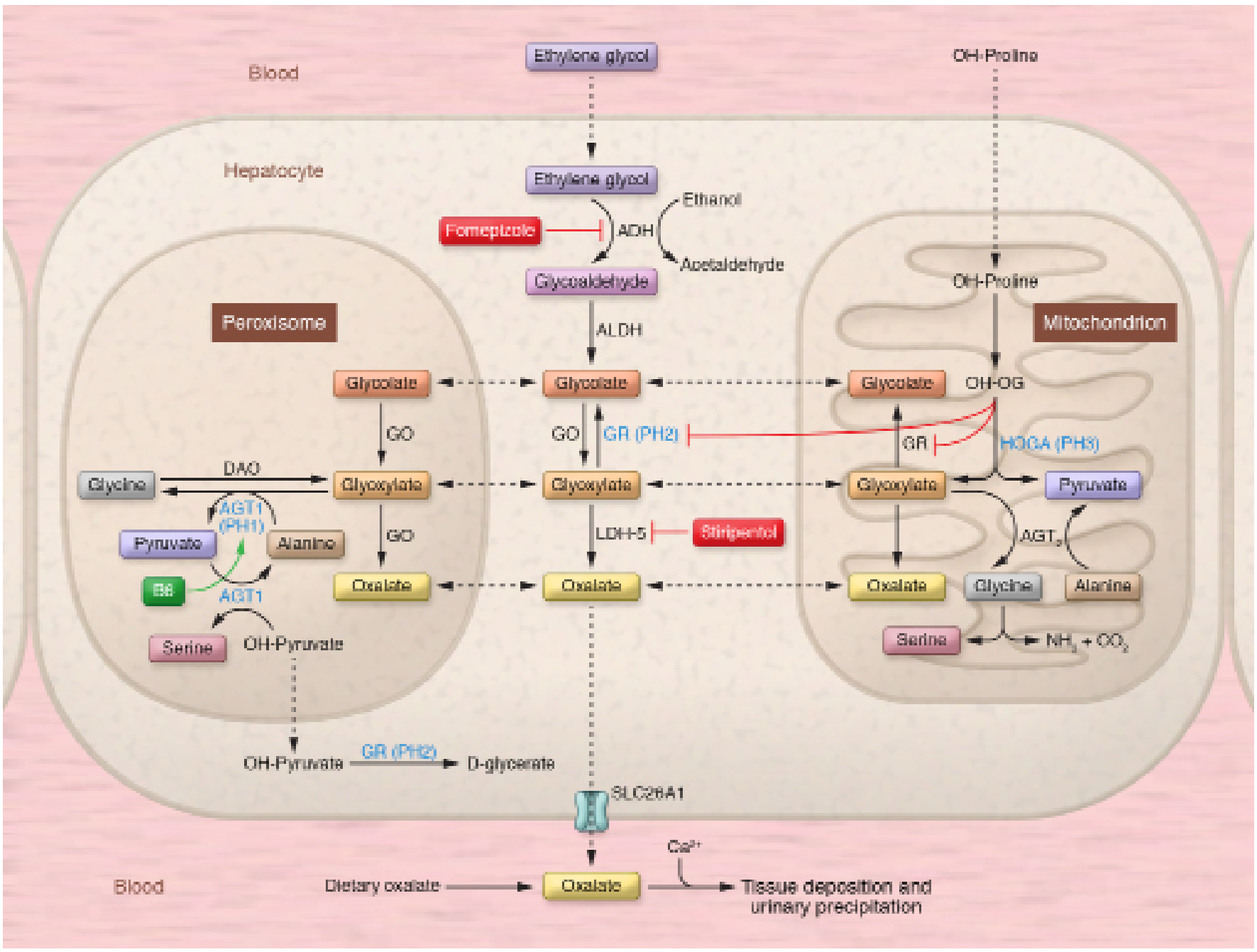

Figure 1. Multicompartment glyoxylate-oxalate metabolism. Multiple metabolic pathways converge to produce glyoxylate before conversion to oxalate. Defects in enzymes responsible for metabolism of glyoxylate and its precursors (denoted in blue) underlie specific forms of primary hyperoxaluria (PH) and lead to accumulation of glyoxylate and consequently to increased oxalate production which leaves the hepatocyte via SLC26a1 on its basolateral membrane. Mutations in AGT1 associate with PH1, mutations in GR are linked to PH2, and HOCA mutations underlie PH3. Inhibition of LDH5 by the drug stiripentol decreases enzymatic conversion of glyoxylate to oxalate and decreases urinary oxalate levels. Vitamin B6 (also known as pyridoxal phosphate) has been shown to increase enzymatic activity and decrease oxalate production in $\mathrm{PH} 1$. $\mathrm{PH}$, primary hyperoxaluria type I; $\mathrm{PH}$, primary hyperoxaluria type II; PH3, primary hyperoxaluria type III; ADH, alcohol dehydrogenase; ALDH, aldehyde dehydrogenase; GR, glycolate reductase; GO, glycolate oxidase; LDH5, lactate dehydrogenase 5; OH-OG, 4-hydroxy-2-oxoglutarate; HOGA, 4-hydroxy-2-oxoglutarate aldolase; AGT1, alanine glyoxylate aminotransferase 1; AGT ${ }_{2}$ alanine glyoxylate aminotransferase 2; DAO, D-amino oxidase; B6, vitamin B6/pyridoxal phosphate.

oxalate is produced as an end-product of metabolism and secreted into the extracellular space by solute-linked carrier $26 \mathrm{a} 1$ (SLC26a1) (6). In the intestine, oxalate is secreted by SLC26a6 but absorbed by passive paracellular diffusion (7). In the kidney, oxalate is freely filtered, and SLC26a1 and SLC26a6 also play a role in tubular secretion and reabsorption of oxalate, resulting in net excretion of less than $0.5 \mathrm{mmol} / \mathrm{d}$ (8). As urine is concentrated through its journey down the nephron, urinary levels of calcium and oxalate rise, and if supersaturation is reached, precipitation and crystallization occur, resulting in both mechanical obstructive tubular injury as well as inflammatory injury from necroptosis that results from indigestible crystals in phagolysosomes. This intratubular and parenchymal $\mathrm{CaOx}$ crystal deposition and the consequent inflammatory reaction can lead to progressive interstitial fibrosis, nephrocalcinosis, and even end-stage renal disease. As the glomerular filtration rate declines, or in the setting of ethylene glycol toxicity, systemic concentrations of oxalate rise and $\mathrm{CaOx}$ levels surpass the supersaturation level systemically, leading to $\mathrm{CaOx}$ deposition in tissues throughout the body.
Dietary hyperoxaluria caused by ingestion of high oxalate foods can be treated with increased calcium intake, which chelates oxalate in the gastrointestinal lumen. Enteric hyperoxaluria results when calcium is instead chelated by free fatty acids as a result of fat malabsorption, thereby leading to a high concentration of free oxalate and increased oxalate absorption. There has been recent interest in the role that the gut microbiome (in particular Oxalobacter formigenes) plays in enteric oxalate degradation, which prevents absorption from ever occurring (9). Additionally, an increase in oxalate 
precursors, such as seen with high doses of ascorbic acid and ethylene glycol, leads to increased oxalate production via metabolism of these precursors (10).

Primary hyperoxaluria $(\mathrm{PH})$ results from three mutations (11). Type I (PH1) results from inactivation of alanine-glyoxylate aminotransferase (AGT1), which prevents glyoxylate from being metabolized to glycine. $\mathrm{PH} 2$ is caused by mutations in glyoxylate reductase (GR), which prevents the breakdown of glyoxylate to glycolate. Finally, in PH3, loss-of-function mutations in 4-hydroxy-2-oxoglutarate aldolase prevent the formation of glyoxylate and pyruvate from 4-hydroxy-2-oxoglutarate, and the subsequent buildup of 4-hydroxy-2-oxoglutarate inhibits the activity of GR, ultimately leading to increased glyoxylate concentration.

The goal of all interventions for hyperoxaluria is to decrease systemic and urinary concentrations of oxalate, thus preventing cocrystallization with calcium. Efforts to reduce dietary oxalate, increase urine volume, and alkalinize the urine with supplemental citrate are the current mainstays of therapy $(11,12)$. Liberalization of dietary calcium and, to a lesser extent, pancreatic enzyme replacement are strategies used in enteric hyperoxaluria. The alcohol dehydrogenase inhibitor fomepizole is often used as an adjunct to hemodialysis in ethylene glycol overdoses and has largely replaced ethanol in these cases (13). In PH1, treatment with the AGT1 cofactor pyridoxal phosphate (vitamin B6) has been shown to increase enzymatic activity and decrease oxalate production (14). There is also research to support a diet low in hydroxyproline for PH2 and PH3 (15). For patients with $\mathrm{PH}$, liver transplantation (or combined liver-kidney if the patient has end-stage renal disease) restores normal oxalate metabolism (11). Unfortunately, for patients with $\mathrm{PH}$ there are few options that are effective for controlling the amount of endogenous hepatic oxalate production.

\section{Conclusions and future directions}

Given these limited treatment options, the introduction of stiripentol as a potential new treatment by Le Dudal et al. is exciting; however, the doses needed are high. As much as $75 \%$ to $90 \%$ of oral stiripentol is rapidly absorbed, with median peak drug concentration achieved in 2-3 hours
(16). $99 \%$ of the drug is protein bound and has a dose-dependent elimination half-life of 4.5 to 13 hours. Stiripentol undergoes hepatic metabolism via demethylenation (CYP1A2, CYP2C19, CYP3A4) and glucuronidation, and these metabolites are excreted in the urine. Because it is both an inhibitor and inducer of CYP1A2, CYP2B6, and CYP3A4, there are important drugdrug interactions to consider. Stiripentol is now approved for use in Dravet syndrome in both the United States and Europe.

Le Dudal et al. used $50 \mathrm{mg} / \mathrm{kg} / \mathrm{d}$ in their patient with $\mathrm{PH} 1$, a dose that represents the upper limit of that recommended for Dravet syndrome. However, the doses of stiripentol used in their animal and in vitro studies exceeded this level by 4 - to 6-fold, raising questions about the generalizability and safety of the potential applications for this therapy. Stiripentol has significant and severe side effects, including somnolence, decreased appetite, weight loss, neutropenia, thrombocytopenia, and suicidal ideation or behavior, when administered in high doses (ref. 17 and drug package insert). An additional challenge will be dealing with the significant CYP inhibition that occurs at higher concentrations. Given these factors, it remains unclear if there will be a role for stiripentol in hyperoxaluric conditions other than in $\mathrm{PH}$ at the recommended doses approved for Dravet syndrome.

What this discovery has done, however, is generate renewed interest in targeted and tailored approaches for the treatment of these disorders. There are ongoing efforts to develop $\mathrm{PH}$ patientspecific therapies, including gene silencing techniques, substrate reduction therapy, and enzyme replacement/substitution therapies $(18,19)$. However, most of these interventions will have roles restricted to patients with rare primary genetic causes rather than the much more common secondary ones. Another research avenue that has garnered recent interest is targeting transcellular oxalate transport via SCL26a1 transporters, as mutations in this gene in humans lead to $\mathrm{CaOx}$ nephrolithiasis (20). Preclinical studies of these and other oxalate transporters are usually performed in knockout mice, which are not the optimum human model for oxalate transport and nephrolithiasis. For instance, initial studies showed a hyper- oxalemic and hyperoxaluric phenotype in Slc26a1 knockout animals (21), whereas more recent knockout studies have not been able to reproduce this phenotype (22), instead highlighting a limited role for SLC26a1 in net oxalate excretion.

The largest unmet need for reducing oxaluria is the case of $\mathrm{CaOx}$ kidney stones, which affect tens of millions of people worldwide. The identification of LDH5 as a potential target raises the hope that this pathway might yield a specific inhibitor that is safe and free of too many drug-drug interactions. One such agent, isosafrole, which has a similar structural backbone as stiripentol but a more potent inhibitory effect on the conversion of pyruvate to lactate in LDH1 and LDH5, resulted in greater suppression of epileptiform activity than stiripentol in vivo (3). It remains to be seen whether small molecules with greater specificity for inhibiting the conversion of pyruvate to lactate will result in a more favorable side effect profile than stiripentol.

While the current study raises some concerns and leaves issues to be addressed, it does renew interest in this pathway and highlights the complicated nature of glyoxylate and oxalate metabolism. Continued efforts in developing novel, targeted therapies may significantly impact public health given the high prevalence of $\mathrm{CaOx}$ nephrolithiasis.

Address correspondence to: Jacob S. Stevens or Qais Al-Awqati, Department of Medicine, Division of Nephrology, Vagelos College of Physicians and Surgeons, Columbia University, 622 W. 168th Street, PH4-124, New York, New York 10032, USA. Phone: 212.305.3273; Email: jss2275@cumc. columbia.edu (JSS). Phone: 212.305.2512; Email: qa1@cumc.columbia.edu (QAA).

1. Le Dudal M, et al. Stiripentol protects against calcium oxalate nephrolithiasis and ethylene glycol poisoning. J Clin Invest. 2019;129(6):2571-2577.

2. Quilichini PP, Chiron C, Ben-Ari Y, Gozlan H. Stiripentol, a putative antiepileptic drug, enhances the duration of opening of GABA-A receptor channels. Epilepsia. 2006;47(4):704-716.

3. Sada N, Lee S, Katsu T, Otsuki T, Inoue T. Epilepsy treatment. Targeting LDH enzymes with a stiripentol analog to treat epilepsy. Science. 2015;347(6228):1362-1367.

4. Magistretti PJ, Allaman I. Lactate in the brain: from metabolic end-product to signalling mole- 
cule. Nat Rev Neurosci. 2018;19(4):235-249.

5. Holmes RP, Assimos DG. Glyoxylate synthesis, and its modulation and influence on oxalate synthesis. JUrol. 1998;160(5):1617-1624.

6. Robijn S, Hoppe B, Vervaet BA, D'Haese PC, Verhulst A. Hyperoxaluria: a gut-kidney axis? Kidney Int. 2011;80(11):1146-1158.

7. Knauf F, et al. Net intestinal transport of oxalate reflects passive absorption and SLC26A6-mediated secretion. J Am Soc Nephrol. 2011;22(12):2247-2255.

8. Knauf F, Velazquez H, Pfann V, Jiang Z, Aronson PS. Characterization of renal $\mathrm{NaCl}$ and oxalate transport in Slc26a6. Am J Physiol Renal Physiol. 2019;316(1):F128-F133.

9. Ticinesi A, et al. Understanding the gut-kidney axis in nephrolithiasis: an analysis of the gut microbiota composition and functionality of stone formers. Gut. 2018;67(12):2097-2106.

10. Jacobsen D, Hewlett TP, Webb R, Brown ST, Ordinario AT, McMartin KE. Ethylene glycol intoxication: evaluation of kinetics and crystal- luria. Am JMed. 1988;84(1):145-152.

11. Cramer SD, Takayama T. Primary hyperoxaluria. In: Lifton RP, Somlo S, Giebisch GH, Seldin DW, eds. Genetic Diseases of the Kidney. 1st ed. San Diego, California, USA: Academic Press; 2009:571-586.

12. Hoppe B, Beck BB, Milliner DS. The primary hyperoxalurias. Kidney Int. 2009;75(12):1264-1271.

13. Brent J. Fomepizole for ethylene glycol and methanol poisoning. N Engl J Med. 2009;360(21):2216-2223.

14. Monico CG, Rossetti S, Olson JB, Milliner DS. Pyridoxine effect in type I primary hyperoxaluria is associated with the most common mutan allele. Kidney Int. 2005;67(5):1704-1709.

15. Fargue S, Milliner DS, Knight J, Olson JB, Lowther WT, Holmes RP. Hydroxyproline metabolism and oxalate synthesis in primary hyperoxaluria. J Am Soc Nephrol. 2018;29(6):1615-1623.

16. Levy RH, Lin HS, Blehaut HM, Tor JA. Pharmacokinetics of stiripentol in normal man: evidence of nonlinearity. J Clin Pharmacol.
1983;23(11-12):523-533.

17. Inoue $Y$, et al. Stiripentol open study in Japanese patients with Dravet syndrome. Epilepsia. 2009;50(11):2362-2368.

18. Dindo M, Conter C, Oppici E, Ceccarelli V, Marinucci L, Cellini B. Molecular basis of primary hyperoxaluria: clues to innovative treatments. Urolithiasis. 2019;47(1):67-78.

19. Weigert A, Martin-Higueras C, Hoppe B. Novel therapeutic approaches in primary hyperoxaluria. Expert Opin Emerg Drugs. 2018;23(4):349-357.

20. Gee HY, et al. Mutations in SLC26A1 cause nephrolithiasis. Am J Hum Genet. 2016;98(6):1228-1234.

21. Dawson PA, et al. Urolithiasis and hepatotoxicity are linked to the anion transporter Sat1 in mice. J Clin Invest. 2010;120(3):706-712.

22. Whittamore JM, Stephens CE, Hatch M. Absence of the sulfate transporter SAT- 1 has no impact on oxalate handling by mouse intestine and does not cause hyperoxaluria or hyperoxalemia. Am J Physiol Gastrointest Liver Physiol. 2019;316(1):G82-G94 